PROCEEDINGS OF THE

AMERICAN MATHEMATICAL SOCIETY

Volume 126, Number 5, May 1998, Pages 1275-1285

S 0002-9939(98)04244-0

\title{
A BORSUK THEOREM FOR ANTIPODAL LINKS AND A SPECTRAL CHARACTERIZATION OF LINKLESSLY EMBEDDABLE GRAPHS
}

\author{
LÁSZLÓ LOVÁSZ AND ALEXANDER SCHRIJVER
}

(Communicated by Jeffry N. Kahn)

\begin{abstract}
For any undirected graph $G$, let $\mu(G)$ be the graph parameter introduced by Colin de Verdière. In this paper we show that $\mu(G) \leq 4$ if and only if $G$ is linklessly embeddable (in $\mathcal{R}^{3}$ ). This forms a spectral characterization of linklessly embeddable graphs, and was conjectured by Robertson, Seymour, and Thomas.

A key ingredient is a Borsuk-type theorem on the existence of a pair of antipodal linked $(k-1)$-spheres in certain mappings $\phi: S^{2 k} \rightarrow \mathcal{R}^{2 k-1}$. This result might be of interest in its own right.

We also derive that $\lambda(G) \leq 4$ for each linklessly embeddable graph $G=$ $(V, E)$, where $\lambda(G)$ is the graph parameter introduced by van der Holst, Laurent, and Schrijver. (It is the largest dimension of any subspace $L$ of $\mathcal{R}^{V}$ such that for each nonzero $x \in L$, the positive support of $x$ induces a nonempty connected subgraph of $G$.)
\end{abstract}

\section{INTRODUCTION}

Motivated by estimating the maximum multiplicity of the second eigenvalue of Schrödinger operators, Colin de Verdière [4] (cf. [5]) introduced an interesting new invariant $\mu(G)$ for graphs $G$, based on spectral properties of matrices associated with $G$. He showed that the invariant is monotone under taking minors (that is, if $H$ is a minor of $G$ then $\mu(H) \leq \mu(G)$ ), that $\mu(G) \leq 1$ if and only if $G$ is a disjoint union of paths, that $\mu(G) \leq 2$ if and only if $G$ is outerplanar, and that $\mu(G) \leq 3$ if and only if $G$ is planar.

In this paper we show that $\mu(G) \leq 4$ if and only if $G$ is linklessly embeddable in $\mathcal{R}^{3}$. An embedding of a graph $G$ in $\mathcal{R}^{3}$ is called linkless if each pair of disjoint circuits in $G$ are unlinked closed curves in $\mathcal{R}^{3}$ (for our ourposes, the following definition of linking suffices: two disjoint curves are unlinked if there is a mapping of the unit disc into $\mathcal{R}^{3}$ such that its boundary is mapped onto the first curve and the image of the disc is disjoint from the second). $G$ is linklessly embeddable if $G$ has a linkless embedding in $\mathcal{R}^{3}$. ('Embedding' presumes that vertices and edges have disjoint images.)

Received by the editors October 15, 1996.

1991 Mathematics Subject Classification. Primary 05C10, 05C50, 57M15; Secondary 05C50, $57 \mathrm{~N} 15$.

Research partially done while visiting the Department of Computer Science at Yale University.

(C)1998 American Mathematical Society 
Our result was conjectured by Robertson, Seymour, and Thomas [9], and has the following context. Robertson, Seymour, and Thomas [10] showed that a graph $G$ is linklessly embeddable if and only if $G$ does not have any of the seven graphs in the Petersen family as a minor - the Petersen family consists of all graphs obtainable from $K_{6}$ by so-called $\Delta \mathrm{Y}$ - and $\mathrm{Y} \Delta$-operations (it includes the Petersen graph). (Y $\Delta$ means deleting a vertex of degree 3, and making its three neighbours mutually adjacent. $\Delta \mathrm{Y}$ is the reverse operation (applied to a triangle).)

Since any of the graphs $G$ in the Petersen family has $\mu(G) \geq 5$ (Bacher and Colin de Verdière [1]), it follows that if $\mu(G) \leq 4$ then $G$ is linklessly embeddable. So we prove the reverse implication. Thus, next to the combinatorial characterization (in terms of minors) of the (topologically defined) class of linklessly embeddable graphs, there is a spectral characterization.

Our proof method also applies to a related parameter called $\lambda(G)$, introduced by van der Holst, Laurent, and Schrijver [7]. It is defined as follows. Let $G=(V, E)$ be a graph. Call a linear subspace $L$ of $\mathcal{R}^{V}$ a representation of $G$ if for each $x \in L$, $\operatorname{supp}_{+}(x)$ is nonempty and $G \mid \operatorname{supp}_{+}(x)$ is a connected graph. Here we use the following notation. If $U \subseteq V$ then $G \mid U$ is the subgraph of $G$ induced by $U$ (and $G-U=G \mid(V \backslash U))$. For any vector $x \in \mathcal{R}^{V}, \operatorname{supp}(x)$ is the support of $x$, that is, $\operatorname{supp}(x):=\{v \in V \mid x(v) \neq 0\}$. The positive support is $\operatorname{supp}_{+}(x):=\{v \in V \mid x(v)>$ $0\}$ and the negative support is $\operatorname{supp}_{-}(x):=\{v \in V \mid x(v)<0\}$.

Now by definition, $\lambda(G)$ is the maximum dimension of any representation $L$ of $G$. It is easy to see that $\lambda(G)$ is monotone under taking minors. Moreover, if $G$ is a clique sum of graphs $G_{1}$ and $G_{2}$ (that is, if $G$ arises from $G_{1}$ and $G_{2}$ by identifying a clique in $G_{1}$ and $\left.G_{2}\right)$, then $\lambda(G)=\max \left\{\lambda\left(G_{1}\right), \lambda\left(G_{2}\right)\right\}$. In [7] it is shown that $\lambda(G) \leq 1$ if and only if $G$ is a forest, that $\lambda(G) \leq 2$ if and only if $G$ is series-parallel, and that $\lambda(G) \leq 3$ if and only if $G$ arises by taking clique sums and subgraphs from planar graphs.

In this paper we show that $\lambda(G) \leq 4$ if $G$ is linklessly embeddable - hence, more generally, if $G$ arises by taking clique sums and subgraphs from linklessly embeddable graphs. We do not know if the reverse implication holds.

A key ingredient in our proof is a Borsuk-type theorem on the existence of antipodal links, which we formulate and prove in Section 2. We derive it from an extension of a theorem of Bajmóczy and Bárány [2] establishing a polyhedral form of Borsuk's antipodal theorem.

In Section 3 we derive that $\lambda(G) \leq 4$ for linklessly embeddable graphs $G$. In Section 4 we give some preliminaries on the Colin de Verdière parameter $\mu(G)$, and after that, in Section 5 , we show that $\mu(G) \leq 4$ for linklessly embeddable graphs $G$. Finally, in Section 6 we consider a number of open questions related to $\mu(G)$ and $\lambda(G)$.

\section{A Borsuk-tyPe THEOREM FOR ANTIPODAL LINKS}

Let $P$ be a convex polytope in $\mathcal{R}^{n}$. We say that two faces $F$ and $F^{\prime}$ are antipodal if there exists a nonzero vector $c$ in $\mathcal{R}^{n}$ such that the linear function $c^{T} x$ is maximized by every point of $F$ and minimized by every point of $F^{\prime}$ (Figure 1 ). So $F$ and $F^{\prime}$ are antipodal if and only if $F-F^{\prime}$ is contained in a face of $P-P$.

Call a continuous map $\phi$ of a cell complex into $\mathcal{R}^{m}$ generic if the images of a $k$-face and an $l$-face intersect only if $k+l \geq m$, and for $k+l=m$ they have a finite 

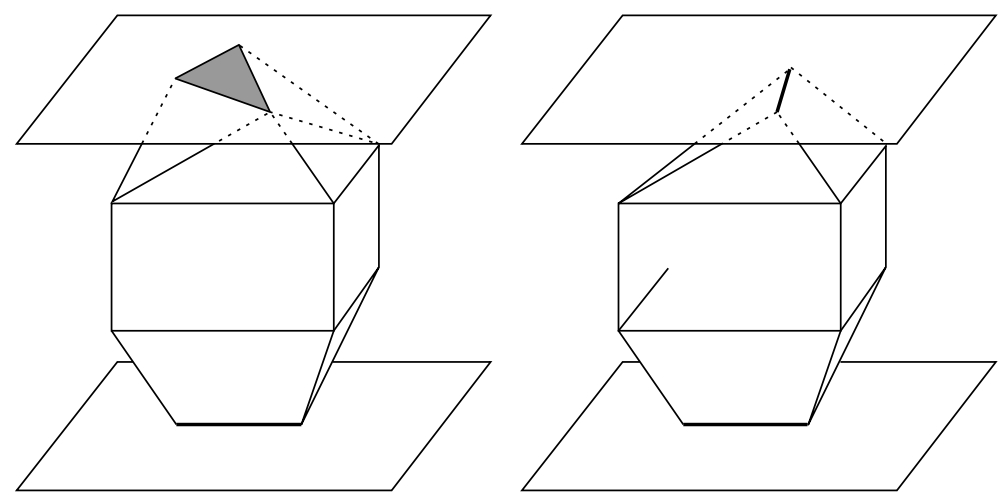

Figure 1. Parallel and non-parallel antipodal faces

number of intersection points, and at these points they intersect transversally. (In this paper, faces are relatively open.)

For any convex polytope $P$ in $\mathcal{R}^{n}$, let $\partial P$ denote its boundary.

The following theorem extends a result of Bajmóczy and Bárány [2]. (The difference is that their theorem concludes that $\phi(F) \cap \phi\left(F^{\prime}\right)$ is nonempty. Their proof uses Borsuk's theorem. We give an independent proof.)

Theorem 1. Let $P$ be a full-dimensional convex polytope in $\mathcal{R}^{n}$ and let $\phi$ be a generic continuous map from $\partial P$ to $\mathcal{R}^{n-1}$. Then there exists a pair of antipodal faces $F$ and $F^{\prime}$ with $\operatorname{dim}(F)+\operatorname{dim}\left(F^{\prime}\right)=n-1$ such that $\left|\phi(F) \cap \phi\left(F^{\prime}\right)\right|$ is odd.

Proof. We prove a more general fact. Call two faces parallel if their projective hulls have a nonempty intersection that is contained in the hyperplane at infinity. So faces $F$ and $F^{\prime}$ are parallel if and only if their affine hulls are disjoint while $F-F$ and $F^{\prime}-F^{\prime}$ have a nonzero vector in common. (Note that two antipodal faces are parallel if $\operatorname{dim}(F)+\operatorname{dim}\left(F^{\prime}\right) \geq n$.)

Now it suffices to show

Let $P$ be a full-dimensional convex polytope in $\mathcal{R}^{n}$ having no parallel faces and let $\phi$ be a generic continuous map from $\partial P$ to $\mathcal{R}^{n-1}$. Then

$$
\sum\left|\phi(F) \cap \phi\left(F^{\prime}\right)\right|
$$

is odd, where the summation extends over all antipodal pairs $\left\{F, F^{\prime}\right\}$ of faces.

(It would be enough to sum over all antipodal pairs $\left\{F, F^{\prime}\right\}$ with $\operatorname{dim}(F)+\operatorname{dim}\left(F^{\prime}\right)=$ $n-1$.)

To see that it suffices to prove (1), it is enough to apply a random projective transformation close to the identity. To be more precise, assume that we have a polytope $P$ that has parallel faces. For every pair $\left(E, E^{\prime}\right)$ of faces whose affine hulls intersect, choose a (finite) point $p_{E E^{\prime}}$ in the intersection of the affine hulls. For every pair $\left(E, E^{\prime}\right)$ of faces whose projective hulls intersect, choose an infinite point $q_{E E^{\prime}}$ in the intersection of their projective hulls. Let $H$ be a finite hyperplane having all the points $p_{E E^{\prime}}$ on one side, and avoiding all the points $q_{F F^{\prime}}$. Apply a projective transformation that maps $H$ onto the hyperplane at infinity, to get a new polytope $P^{\prime}$. It is clear that $P^{\prime}$ has no parallel faces, and it is easy to argue 
that every pair of faces that are antipodal in $P^{\prime}$ correspond to antipodal faces in $P$. Hence (1) implies the theorem.

We now prove (1). Let $P$ be a convex polytope in $\mathcal{R}^{n}$ having no parallel faces. For any two faces $F, F^{\prime}$, write $F \leq F^{\prime}$ if $F \subseteq \overline{F^{\prime}}$. Then:

(i) if $A$ and $B$ are antipodal faces, then $A-B$ is a face of $P-P$, with $\operatorname{dim}(A-B)=$ $\operatorname{dim}(A)+\operatorname{dim}(B)$

(ii) if $F$ is a face of $P-P$, then there exists a unique pair $A, B$ of antipodal faces with $A-B=F$;

(iii) for any two pairs $A, B$ and $A^{\prime}, B^{\prime}$ of antipodal faces one has $A-B \leq A^{\prime}-B^{\prime}$ if and only if $A \leq A^{\prime}$ and $B \leq B^{\prime}$.

This gives the following observation:

For every pair of faces $A$ and $B$ with $\operatorname{dim}(A)+\operatorname{dim}(B)=$ $n-2$, the number of antipodal pairs $\left\{F, F^{\prime}\right\}$ of faces with $A \leq F$ and $B \leq F^{\prime}$ and $\operatorname{dim}(F)+\operatorname{dim}\left(F^{\prime}\right)=n-1$ is 0

$$
\text { or } \overline{2} \text {. }
$$

To see (3), it is clear that if $A$ and $B$ are not antipodal, then this number is 0 . Suppose that they are antipodal. Then the number is 2 , since by (2), it is equal to the number of facets of $P-P$ incident with the $(n-2)$-face $A-B$.

To prove (1), we use a "deformation" argument. The statement is true for the following mapping $\phi$ : pick a point $q$ very near the center of gravity of some facet $F$ (outside $P$ ), and project $\partial P$ from $q$ onto the hyperplane $H$ of $F$. Then the only nontrivial intersection is that the image of the (unique) vertex of $P$ farthest from $H$ is contained in $F$.

Now we deform this map to $\phi$. We may assume that the images of two faces $E$ and $E^{\prime}$ with $\operatorname{dim}(E)+\operatorname{dim}\left(E^{\prime}\right) \leq n-3$ never meet; but we have to watch when $\phi(A)$ passes through $\phi(B)$, where $A$ and $B$ are faces with $\operatorname{dim}(A)+\operatorname{dim}(B)=n-2$. But then $\left|\phi(F) \cap \phi\left(F^{\prime}\right)\right|$ changes exactly when $A \subseteq F$ and $B \subseteq F^{\prime}$. By (3), this does not change the parity. This proves (1), and hence the theorem.

For any polytope $P$, let $(P)_{k}$ denote its $k$-skeleton. Two disjoint images $A$ and $B$ of $(d-1)$-spheres in $\mathcal{R}^{2 d-1}$ are said to have an odd linking number if the image of $A$ can be extended to the image of a $d$-ball with an odd number of transveral intersections with the image of $B$ (and no other intersections). So having an odd linking number implies being linked.

Corollary 1.1. Let $P$ be a full-dimensional convex polytope in $\mathcal{R}^{2 k+1}$ and let $\phi$ be an embedding of $(P)_{k-1}$ into $\mathcal{R}^{2 k-1}$. Then there exists a pair of antipodal $k$-faces $F$ and $F^{\prime}$ such that $\phi(\partial F)$ and $\phi\left(\partial F^{\prime}\right)$ have an odd linking number.

Proof. First we extend $\phi$ with a last coordinate equal to 0 , to obtain an embedding $\psi$ of $(P)_{k-1}$ into $\mathcal{R}^{2 k}$. Next we extend $\psi$ to a generic mapping $\partial P \rightarrow \mathcal{R}^{2 k}$, in such a way that $\psi(x)$ has last coordinate positive if $x \in \partial P \backslash(P)_{k-1}$.

By Theorem 1, $P$ has two antipodal faces $F$ and $F^{\prime}$ such that $\operatorname{dim}(F)+\operatorname{dim}\left(F^{\prime}\right)=$ $2 k$ and $\left|\psi(F) \cap \psi\left(F^{\prime}\right)\right|$ is odd. If $\operatorname{dim}(F) \leq k-1$, then the last coordinate of each point in $\psi(F)$ is 0 , while the last coordinate of each point in $\psi\left(F^{\prime}\right)$ is positive (as $\operatorname{dim}\left(F^{\prime}\right) \geq k$ ). So $\operatorname{dim}(F) \geq k$, and similarly $\operatorname{dim}\left(F^{\prime}\right) \geq k$. Therefore, $\operatorname{dim}(F)=$ $\operatorname{dim}\left(F^{\prime}\right)=k$.

Then the boundaries of $F$ and $F^{\prime}$ are $(k-1)$-spheres $S_{1}$ and $S_{2}$, mapped disjointly into $\mathcal{R}^{2 k-1}$, and the mappings extend to mappings of the $k$-balls into the "upper" 
halfspace of $\mathcal{R}^{2 k}$, so that the images of the balls intersect at an odd number of points. But this implies that the images of the spheres are linked. In fact, if they are not linked, then there exists an extension of the map of $\partial F$ to a continuous mapping $\psi^{\prime}$ of $F$ into $\mathcal{R}^{2 k}$ such that the image of every point in the interior of $F$ has last coordinate equal to 0 , and $\psi^{\prime}(F)$ intersects $\psi\left(\partial F^{\prime}\right)$ transversally in an even number of points. We can extend the map of $\partial F^{\prime}$ to a continuous mapping $\psi^{\prime}$ of $F^{\prime}$ into $\mathcal{R}^{2 k}$ such that the image of every point in the interior of $F$ has a negative last coordinate 0 . Then we get two maps of the $k$-sphere into $\mathcal{R}^{2 k}$ with an odd number of transversal intersection points, which is impossible. This contradiction completes the proof.

\section{3. $\lambda(G) \leq 4$ FOR LINKLESSLY EMBEDDABLE GRAPHS $G$}

In this section we focus on the graph parameter $\lambda(G)$ introduced in [7], and show that $\lambda(G) \leq 4$ for linklessly embeddable graphs. The proof method also serves as an introduction to the methods used in proving that $\mu(G) \leq 4$ for linklessly embeddable graphs. We gave the definition of $\lambda(G)$ in Section 1.

Theorem 2. If $G$ is linklessly embeddable, then $\lambda(G) \leq 4$.

Proof. Let $G$ be linklessly embedded in $\mathcal{R}^{3}$, and suppose that $\lambda(G) \geq 5$. Then there is a 5-dimensional subspace $L$ of $\mathcal{R}^{V}$ such that $G \mid \operatorname{supp}_{+}(x)$ is nonempty and connected for each nonzero $x \in L$.

Call two elements $x$ and $x^{\prime}$ of $L$ equivalent if $\operatorname{supp}_{+}(x)=\operatorname{supp}_{+}\left(x^{\prime}\right)$ and $\operatorname{supp}_{-}(x)$ $=\operatorname{supp}_{-}\left(x^{\prime}\right)$. The equivalence classes decompose $L$ into a centrally symmetric complex $\mathcal{P}$ of pointed polyhedral cones. Choose a sufficiently dense set of vectors of unit length from every cone in $\mathcal{P}$, in a centrally symmetric fashion, and let $P$ be the convex hull of these vectors. Then $P$ is a 5 -dimensional centrally symmetric convex polytope such that every face of $P$ is contained in a cone of $\mathcal{P}$.

We define an embedding $\phi$ of $(P)_{1}$ in $\mathcal{R}^{3}$. For each vertex $v$ of $P$, we choose a node $v^{\prime}$ in $\operatorname{supp}_{+}(v)$, end we let $\phi(x)$ be a point in $\mathcal{R}^{3}$ very near $v^{\prime}$. For each edge $e=u v$ of $P$, we choose a path $e^{\prime}$ connecting $u^{\prime}$ and $v^{\prime}$ in $G \mid \operatorname{supp}_{+}(x)$, where $x$ is an interior point of $e$. (By our construction, $\operatorname{supp}_{+}(x)$ is independent of the choice of $x$, and contains both $\operatorname{supp}_{+}(u)$ and $\operatorname{supp}_{+}(v)$.) Then we map $e$ onto a Jordan curve connecting $\phi(u)$ and $\phi(v)$ very near $e^{\prime}$. Clearly we can choose the images of the vertices and edges so that this map $\phi$ is one-to-one (Figure 2).

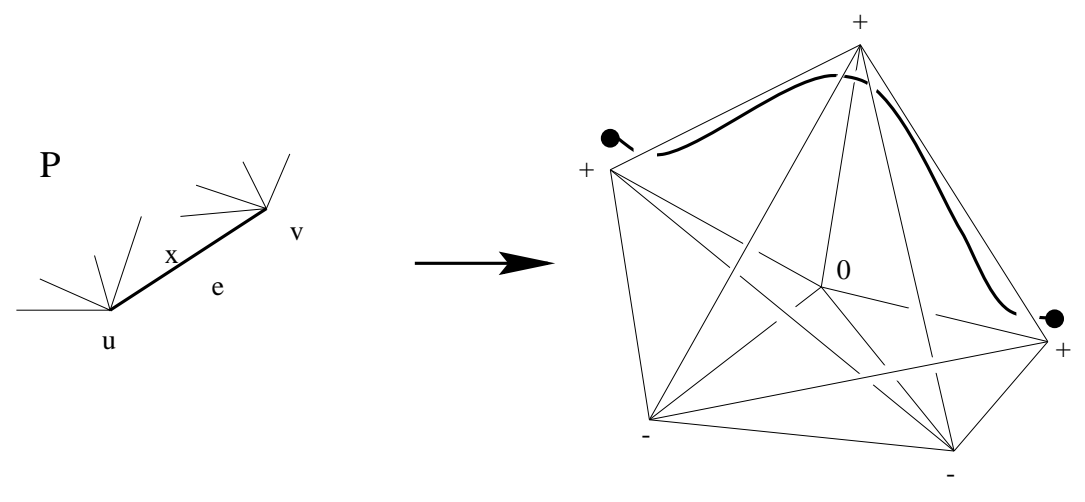

Figure 2. Constructing an embedding of $(P)_{1}$ 
Then by Corollary 1.1, $P$ has two antipodal 2-faces $F$ and $F^{\prime}$ such that the images of their boundaries are linked. Since $P$ is centrally symmetric, there is a facet $D$ of $P$ such that $F \subseteq \bar{D}$ and $F^{\prime} \subseteq-\bar{D}$. Let $y$ be a vector in the interior of $D$. Then the images of $\partial F$ and $\partial F^{\prime}$ are very near subgraphs spanned by $\operatorname{supp}_{+}(y)$ and supp_ $(y)$, respectively, and hence some cycle of $G$ spanned by $\operatorname{supp}_{+}(y)$ must be linked with some cycle in $\operatorname{supp}_{-}(y)$, a contradiction.

Corollary 2.1. If $G$ is obtained from linklessly embedded graphs by taking clique sums and subgraphs, then $\lambda(G) \leq 4$.

Proof. Directly from Theorem 2 and the fact that the class of graphs $G$ with $\lambda(G) \leq$ 4 is closed under taking clique sums and subgraphs ([7]).

\section{The Colin de Verdière Parameter $\mu(G)$}

We now go over to the Colin de Verdière parameter $\mu(G)$, for which we first give some background.

Let $G=(V, E)$ be an undirected graph, which we assume without loss of generality to have vertex set $\{1, \ldots, n\}$. Then $\mu(G)$ is the largest corank of any symmetric real-valued $n \times n$ matrix $M=\left(m_{i, j}\right)$ satisfying:

(i) $\quad M$ has exactly one negative eigenvalue, of multiplicity 1 ,

(ii) for all $i, j$ with $i \neq j, m_{i, j}<0$ if

$i$ and $j$ are adjacent, and $m_{i, j}=0$ if

$i$ and $j$ are nonadjacent,

(iii) there is no nonzero symmetric $n \times n$ matrix $X=\left(x_{i, j}\right)$ such that $M X=0$ and such that $x_{i, j}=0$ whenever $i=j$ or $m_{i, j} \neq 0$.

There is no condition on the diagonal entries $m_{i, i}$. (The corank $\operatorname{corank}(M)$ of a matrix $M$ is the dimension of its kernel.) Condition 4(iii) is called the strong Arnold property (or strong Arnold hypothesis).

There exist matrices $M$ satisfying (4), for which $\operatorname{ker}(M)$ is a not a representation of $G$; that is, for which there exist $x \in \operatorname{ker}(M)$ with $G \mid \operatorname{supp}_{+}(x)$ disconnected. (Otherwise $\mu(G) \leq \lambda(G)$ would follow, which is not true.) The Petersen graph provides an example. Let $A$ be the adjacency matrix of the Petersen graph and let $M=I-A$. Let $e$ and $e^{\prime}$ be two edges at distance 2, and define a vector $q_{e e^{\prime}} \in \mathcal{R}^{V}$ as 1 on the endnodes of $e,-1$ on the endnodes of $e^{\prime}$, and 0 elsewhere. Then $q_{e e^{\prime}} \in \operatorname{ker}(M)$, and it is easy to see that in fact $\operatorname{ker}(M)$ is generated by these vectors. Now if $e, e^{\prime}$ and $e^{\prime \prime}$ are three edges that are mutually at distance 2, then $q_{e e^{\prime}}+q_{e e^{\prime \prime}}$ is a vector in $\operatorname{ker}(M)$ with $\operatorname{supp}_{-}(q)$ having two components (Figure 3 ). We shall see that this is as bad as it ever gets.

The following lemma extends a lemma in [6]; the methods we use to prove it are close to those used in [8].

For any graph $G=(V, E)$ and $U \subseteq V$, let $N(U)$ be the set of vertices in $V \backslash U$ that are adjacent to at least one vertex in $U$. For any $V \times V$ matrix and $I, J \subseteq V$, let $M_{I \times J}$ denote the submatrix induced by the rows in $I$ and columns in $J$, and let $M_{I}:=M_{I \times I}$. For any vector $z \in \mathcal{R}^{I}$ and $J \subseteq I$, let $z_{J}$ be the subvector of $z$ induced by the indices in $J$.

Lemma 1. Let $G$ be a connected graph, let $M$ be a matrix satisfying (4), and let $x$ be a vector in $\operatorname{ker}(M)$ with $G \mid \operatorname{supp}_{+}(x)$ disconnected. Then there are no edges 

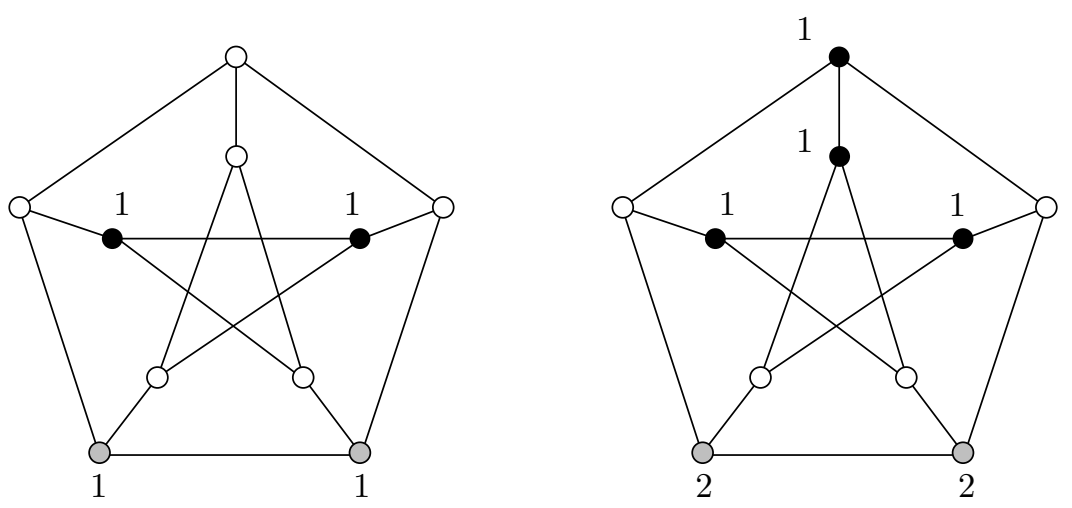

Figure 3. A vector on the Petersen graph with disconnected positive support

connecting $\operatorname{supp}_{+}(x)$ and $\operatorname{supp}_{-}(x)$, and each component $K$ of $G \mid \operatorname{supp}(x)$ satisfies $N(K)=N(\operatorname{supp}(x))$.

Proof. Let $z$ be a positive eigenvector of $M$ (by the Perron-Frobenius theorem, $z$ is unique up to scaling, and belongs to the smallest eigenvalue of $M$ ).

Let $I$ and $J$ be two components of $G \mid \operatorname{supp}_{+}(x)$. Let $L:=\operatorname{supp}_{-}(x)$. As $M x=0$, we have

$$
\begin{aligned}
& M_{I \times I} x_{I}+M_{I \times L} x_{L}=0, \\
& M_{J \times J} x_{J}+M_{J \times L} x_{L}=0 .
\end{aligned}
$$

Let $\lambda:=z_{I}^{T} x_{I} / z_{J}^{T} x_{J}$. Define $y \in \mathcal{R}^{V}$ by: $y_{i}:=x_{i}$ if $i \in I, y_{i}:=-\lambda x_{i}$ if $i \in J$, and $y_{i}:=0$ if $i \notin I \cup J$. Then $z^{T} y=z_{I}^{T} x_{I}-\lambda z_{J}^{T} x_{J}=0$. Moreover, one has (since $\left.M_{I \times J}=0\right)$

$$
\begin{aligned}
& y^{T} M y=y_{I}^{T} M_{I} y_{I}+y_{J}^{T} M_{J} y_{J}=x_{I}^{T} M_{I} x_{I}+\lambda^{2} x_{J}^{T} M_{J} x_{J}= \\
& -x_{I}^{T} M_{I \times L} x_{L}-\lambda^{2} x_{J}^{T} M_{J \times L} x_{L} \leq 0
\end{aligned}
$$

(using (5)), since $M_{I \times L}$ and $M_{J \times L}$ are nonpositive, and since $x_{I}>0, x_{J}>0$ and $x_{L}<0$.

Now $z^{T} y=0$ and $y^{T} M y \leq 0$ imply that $M y=0$ (as $M$ is symmetric and has exactly one negative eigenvalue, with eigenvector $z$ ). Therefore, $y \in \operatorname{ker}(M)$. By 4(i), for distinct $u, v$, entry $M_{u, v}$ of $M$ is negative if and only if $u$ and $v$ are adjacent. This implies that each vertex in $V \backslash \operatorname{supp}(y)$ adjacent to $\operatorname{supp}_{+}(y)=I$ is also adjacent to $\operatorname{supp}_{-}(y)=J$, and conversely; that is, $N(I)=N(J)$.

Also $x-y$ belongs to $\operatorname{ker}(M)$. Hence, again, any vertex $v \in V \backslash \operatorname{supp}(x-y)$ is adjacent to $\operatorname{supp}_{-}(x-y)$ if and only if $v$ is adjacent to $\operatorname{supp}_{+}(x-y)$. As $\operatorname{supp}_{+}(x-y)=\operatorname{supp}_{+}(x) \backslash I$ and as $I$ is a component of $G \mid \operatorname{supp}_{+}(x)$, no vertex in $I$ is adjacent to $\operatorname{supp}_{+}(x-y)$. Hence no vertex in $I$ is adjacent to $\operatorname{supp}_{-}(x-y)=$ $\operatorname{supp}_{-}(x)$. As $I$ is an arbitrary component of $G \mid \operatorname{supp}_{+}(x)$, it follows that there is no edge connecting $\operatorname{supp}_{+}(x)$ and $\operatorname{supp}_{-}(x)$. So each component of $G \mid \operatorname{supp}(x)$ is a component of either $G \mid \operatorname{supp}_{+}(x)$ or $G \mid \operatorname{supp}_{-}(x)$.

Since $N(I)=N(J)$ for any two components $I, J$ of $G \mid \operatorname{supp}_{+}(x)$, and similarly, $N(I)=N(J)$ for any two components $I, J$ of $G \mid \operatorname{supp}_{-}(x)$, and since $N\left(\operatorname{supp}_{+}(x)\right)$ 
$=N\left(\operatorname{supp}_{-}(x)\right)$, we have that

$$
N(I)=N(\operatorname{supp}(x))
$$

for any component $I$ of $G \mid \operatorname{supp}(x)$.

$$
\text { 5. } \mu(G) \leq 4 \text { FOR LINKLESSLY EMBEDDABLE GRAPHS }
$$

Theorem 3. A graph $G$ is linklessly embeddable if and only if $\mu(G) \leq 4$.

Proof. By the results of [10] and [1] it suffices to show that $\mu(G) \leq 4$ if $G$ is linklessly embeddable.

Let $G$ be linklessly embeddable and suppose that $\mu(G) \geq 5$. By the results of [10] we may assume that $G$ is 'flatly embedded' in $\mathcal{R}^{3}$; that is, for each circuit $C$ in $G$ there exists an open disk ('panel') $D$ in $\mathcal{R}^{3}$ with the property that $D$ is disjoint from $G$ and has boundary $C$. (Any flat embedding is also linkless, but the reverse is generally not true. But in [10] it has been shown that if $G$ has a linkless embedding it also has a flat embedding.)

We take a counterexample $G$ with a minimum number of vertices. Then $G$ is 4-connected. For suppose that $G$ has a minimum-size vertex cut $U$ with $|U| \leq 3$. Consider any component $K$ of $G-U$. Then the graph $G^{\prime}$ obtained from $G-K$ by adding a clique on $U$ is a linkless embeddable graph again, because, if $|U| \leq 2$, $G^{\prime}$ is a minor of $G$, and if $|U|=3, G^{\prime}$ can be obtained from a minor of $G$ by a Y $\Delta$-operation. As $G^{\prime}$ has fewer vertices than $G$, we have $\mu\left(G^{\prime}\right) \leq 4$. As this is true for each component $K, G$ is a a subgraph of a clique sum of graphs $G^{\prime}$ with $\mu\left(G^{\prime}\right) \leq 4$, with cliques of size at most 3 , and hence by the results of $[8], \mu(G) \leq 4$.

Let $M$ be a matrix satisfying (4) with $\operatorname{corank}(M)=5$. We proceed as in the proof of Theorem 2. Call two elements $x$ and $x^{\prime}$ of $\operatorname{ker}(M)$ equivalent if $\operatorname{supp}_{+}(x)=$ $\operatorname{supp}_{+}\left(x^{\prime}\right)$ and $\operatorname{supp}_{-}(x)=\operatorname{supp}_{-}\left(x^{\prime}\right)$. The equivalence classes decompose $\operatorname{ker}(M)$ into a centrally symmetric complex $\mathcal{P}$ of pointed polyhedral cones. Call a cone $f$ of $\mathcal{P}$ broken if $G \mid \operatorname{supp}_{+}(x)$ is disconnected for any $x \in f$.

To study broken cones, we first remark:

for each $x \in \operatorname{ker}(M)$ with $G \mid \operatorname{supp}_{+}(x)$ disconnected, $G \mid \operatorname{supp}(x)$ has exactly three components, say $K_{1}, K_{2}$, and $K_{3}$, with $K_{1} \cup K_{2}=\operatorname{supp}_{+}(x)$ and $K_{3}=\operatorname{supp}_{-}(x)$, and with $N\left(K_{i}\right)=V \backslash \operatorname{supp}(x)$ for $i=1,2,3$.

This follows directly from Lemma 1 , using the 4-connectivity of $G$ and the fact that $G$ has no $K_{4,4}$-minor (as $K_{4,4}$ is not linklessly embeddable (cf. [10])).

Now (7) gives:

any broken cone $f$ is 2 -dimensional.

Indeed, choose $x \in f$, and let $K_{1}, K_{2}$, and $K_{3}$ be as in (7). Consider any $y \in f$. As $\operatorname{supp}(y)=\operatorname{supp}(x)$, we have that $M_{K_{i}} y_{K_{i}}=0$ for $i=1,2,3$. As $M_{K_{i}} x_{K_{i}}=0$ and as $x_{K_{i}}$ is fully positive or fully negative, we know by the Perron-Frobenius theorem that $y_{K_{i}}=\lambda_{i} x_{K_{i}}$ for some $\lambda_{i}>0(i=1,2,3)$. Moreover, for the positive eigenvector $z$ of $M$ we have that $z^{T} y=z^{T} x=0$. Conversely, any vector $y \in \mathcal{R}^{V}$ with $z^{T} y=0$ and $\operatorname{supp}(y)=\operatorname{supp}(x)$ and for which there exist $\lambda_{1}, \lambda_{2}, \lambda_{3}>0$ with $y_{K_{i}}=\lambda_{i} x_{K_{i}}$ for $i=1,2,3$, belongs to $f$, since it belongs to $\operatorname{ker}(M)$. This follows from the fact that $z^{T} y=0$ and $y^{T} M y=0$. So $f$ is 2-dimensional, proving (8).

Now choose a sufficiently dense set of vectors of unit length from every cone in $\mathcal{P}$, in a centrally symmetric fashion, and let $P$ be the convex hull of these vectors. 
Then $P$ is a 5 -dimensional centrally symmetric convex polytope such that every face of $P$ is contained in a cone of $\mathcal{P}$. We choose the vectors densely enough such that every face of $P$ contains at most one edge that is part of a 2 -dimensional cone in $\mathcal{P}$. We call an edge of $P$ broken if it is contained in a broken cone in $\mathcal{P}$.

We define an embedding $\phi$ of the 1 -skeleton $(P)_{1}$ of $P$ in $\mathcal{R}^{3}$. We map each vertex $x$ of $P$ to a point $\phi(x)$ near supp $(x)$, and we map any unbroken edge $e=x y$ of $P$ to a curve connecting $\phi(x)$ and $\phi(y)$ near $G \mid \operatorname{supp}_{+}(z)$, where $z \in e$. We do this in such a way that the mapping is one-to-one.

Consider next a broken edge $e$ of $P$. Choose $x \in e$, let $K_{1}, K_{2}$, and $K_{3}$ be as in (7), and let $T:=N(\operatorname{supp}(x))$. Then

there is a curve $C$ in $\mathcal{R}^{3} \backslash G$ connecting $K_{1}$ and $K_{2}$ such that there is no pair of disjoint linked circuits $A$ in $G \mid\left(K_{1} \cup K_{2} \cup T\right) \cup C$ and $B$ in $G \mid\left(K_{3} \cup T\right)$.

To see this, let $H$ be the flatly embedded graph obtained from $G$ by contracting $K_{i}$ to one vertex $v_{i}(i=1,2,3)$. It suffices to show that there is a curve $C$ connecting $v_{1}$ and $v_{2}$ such that the graph $H \cup C$ is linklessly embedded. (Indeed, having $C$ with $H \cup C$ linklessly embedded, we can decontract each $K_{i}$ slightly, and make $C$ connect two points in $K_{1}$ and $K_{2}$. Consider a circuit $A$ in $G \mid\left(K_{1} \cup K_{2} \cup T\right) \cup C$ and a circuit $B$ in $G \mid\left(K_{3} \cup T\right)$ disjoint from $A$. Contracting $K_{1}, K_{2}$, and $K_{3}$, we obtain disjoint cycles $A^{\prime}$ and $B^{\prime}$ in $H \cup C$. (A cycle is an edge-disjoint union of circuits.) As $H \cup C$ is linklessly embedded, $A^{\prime}$ and $B^{\prime}$ are unlinked. Hence $A$ and $B$ are unlinked.)

Now $H \mid T$ is a Hamiltonian circuit on $T$, or part of it. Otherwise, $H \mid T$ would contain, as a minor, a graph on four vertices that is either a $K_{1,3}$ or a triangle with an isolated vertex. In both cases, it implies that $H$ has a minor in the Petersen family, which is not possible since $H$ is linklessly embedded.

So $H$ is isomorphic to the complete bipartite graph $K_{3,|T|}$, with some edges on $T$ added forming part (or all) of a Hamiltonian circuit on $T$. As $H$ is flatly embedded, for each edge $t_{1} t_{2}$ of $H \mid T$ there is an open disk ("panel") with boundary the triangle $t_{1} t_{2} v_{3}$, in such a way that the panels are pairwise disjoint (by Böhme's lemma [3] (cf. [11], [10])). Since the union of $H \mid\left(K_{3} \cup T\right)$ with the panels is contractible, there is a curve $C$ from $v_{1}$ to $v_{2}$ not intersecting any panel. This curve has the required properties, showing (9).

We now define $\phi$ on $e$ close to a curve in $G \mid\left(K_{1} \cup K_{2}\right) \cup C$, again so that it is one-to-one. We do this for each broken edge $e$, after which the construction of $\phi$ is finished.

Then by Corollary 1.1, there are two antipodal 2-faces $F$ and $F^{\prime}$ such that the images of their boundaries are linked. Since $P$ is centrally symmetric, there is a facet $D$ of $P$ such that $F \subseteq \bar{D}$ and $F^{\prime} \subseteq-\bar{D}$. Let $y$ be a vector in the interior of $D$. Then $\partial F$ and $\partial F^{\prime}$ have image in $\operatorname{supp}_{+}(y)$ and $\operatorname{supp}_{-}(y)$ respectively. If $\partial F$ and $\partial F^{\prime}$ do not contain any broken edge, then it would follow that $G$ has two disjoint linked circuits - a contradiction.

So we can assume that $\partial F$ contains a broken edge $e$. Then it is the only broken edge in $\partial F$, since by our construction, $\partial D$ contains at most one edge of $P$ that is part of a 2-dimensional cone $f$ in $\mathcal{P}$. So $f$ is broken. Moreover, $\partial F^{\prime}$ does not contain any broken edge. For suppose that $\partial F^{\prime}$ contains broken edge $e^{\prime}$ of $P$. Then $e^{\prime}$ is part of a broken 2-dimensional cone $f^{\prime}$ in $\mathcal{P}$, and hence $f^{\prime}=-f$ (since $D$ is incident with at most one edge that is part of a 2 -dimensional cone of $\mathcal{P}$ ). However, 
as $f$ is broken, $-f$ is not broken, since $G \mid \operatorname{supp}_{-}(x)$ is connected for any $x \in f$ (by $7)$.

Choose $x \in f$, and consider the partition of $V$ into $K_{1}, K_{2}, K_{3}$, and $T$ as above, with $\operatorname{supp}_{+}(x)=K_{1} \cup K_{2}$ and $\operatorname{supp}_{-}(x)=K_{3}$. Then $K_{1} \cup K_{2} \subseteq \operatorname{supp}_{+}(y)$ and $K_{3} \subseteq \operatorname{supp}_{-}(y)$, and hence $\operatorname{supp}_{+}(y) \subseteq K_{1} \cup K_{2} \cup T$ and supp_ $(y) \subseteq K_{3} \cup T$. So the image of $\partial F$ is close to $G \mid\left(K_{1} \cup K_{2} \cup T\right) \cup C$, where $C$ is the curve constructed for the broken edge $e$ of $P$, and the image of $\partial F^{\prime}$ is close to $G \mid\left(K_{3} \cup T\right)$. This contradicts (9).

Note that in this proof, the strong Arnold property is hardly used. Also the lemma remains true without the strong Arnold property. In fact, the above shows that for a 4-connected linklessly embeddable graph $G$, each matrix $M$ satisfying (4)(i) and (ii) has corank $(M) \leq 4$.

\section{SOME OPEN QUESTIONS}

A first question that comes up is whether one can prove that a graph $G$ is linklessly embeddable if $\mu(G) \leq 4$ directly, that is, without using the RobertsonSeymour-Thomas theorem. In other words, does the nonexistence of a matrix $M$ of corank 5 satisfying (4) imply, in a direct way, the existence of a linkless embedding?

Secondly, the above does not complete the characterization of graphs $G$ satisfying $\lambda(G) \leq 4$. Each graph $G$ obtainable from linklessly embeddable graphs by taking clique sums and subgraphs satisfies $\lambda(G) \leq 4$, but it is an open question if the reverse also holds. To answer this question, one might investigate the class of minor-minimal graphs that cannot be obtained from linklessly embeddable graphs by taking clique sums and subgraphs. It is not known what the full list of these graphs is.

In [7] the following graphs are shown to be minor-minimal with respect to the property $\lambda(G) \geq 5$. First $G=K_{6}$ (all other graphs $G$ in the Petersen family satisfy $\lambda(G) \leq 4)$. Next, consider the graph $V_{8}$ with vertices $v_{1}, \ldots, v_{8}$, with $v_{i}$ and $v_{j}$ adjacent if and only if $|i-j| \in\{1,4,7\}$. (It was shown by Wagner [12] that a graph $G$ can be obtained from planar graphs by taking clique sums and subgraphs if and only if $G$ has no $K_{5^{-}}$or $V_{8}$-minor. So $K_{5}$ and $V_{8}$ are the only minor-minimal graphs $G$ with $\lambda(G) \geq 4$.)

Let $V_{9}^{\prime}$ arise from $V_{8}$ by adding an extra vertex $v_{0}$, adjacent to $v_{2}, v_{4}, v_{6}, v_{7}$, $v_{8}$. Similarly, let $V_{9}^{\prime \prime}$ arise from $V_{8}$ by adding an extra vertex $v_{0}$ adjacent to $v_{2}$, $v_{3}, v_{5}, v_{7}, v_{8}$. It is shown in [7] that $V_{9}^{\prime}$ and $V_{9}^{\prime \prime}$ are minor-minimal graphs $G$ with $\lambda(G) \geq 5$.

The graphs $V_{9}^{\prime}$ and $V_{9}^{\prime \prime}$ are also minor-minimal graphs not obtainable from linklessly embeddable graphs by taking clique sums and subgraphs. This can be seen as follows. Since $\lambda\left(V_{9}^{\prime}\right)=\lambda\left(V_{9}^{\prime \prime}\right)=5$, it follows from Corollary 2.1 that these two graphs indeed are not obtainable in such a way. Moreover, to see that they are minor-minimal, observe that deleting or contracting any edge of $V_{9}^{\prime}$ or $V_{9}^{\prime \prime}$ produces a graph that has a vertex whose deletion makes the graph a clique sum of planar graphs.

Since the class of graphs $G$ with $\lambda(G) \leq 4$ is closed under taking $\Delta Y$ operations (not under $\mathrm{Y} \Delta$ ), we can obtain other graphs with $\lambda(G) \geq 5$ by applying a Y $\Delta$ operation to $V_{9}^{\prime}$ or $V_{9}^{\prime \prime}$. Any of them contains a $K_{6}$-minor, except if we apply Y $\Delta$ to vertex $v_{1}$ (or equivalently, to $v_{5}$ ) of $V_{9}^{\prime}$. 
So it could be true that $\lambda(G) \leq 4$ if and only if $G$ is obtainable from linklessly embeddable graphs by taking clique sums and subgraphs.

Another open question is whether $\lambda(G) \leq \mu(G)$ for each graph $G$. That is, for any representation $L$ of $G$ is there a matrix $M$ satisfying 4 with $\operatorname{dim}(L) \leq \operatorname{corank}(M)$ ? This is true if $\mu(G) \leq 4$.

In fact, a tempting, more general speculation is that for any natural number $t$ :

(???) a graph $G$ satisfies $\lambda(G) \leq t$ if and only if $G$ is obtainable from graphs $H$ satisfying $\mu(H) \leq t$ by taking clique sums and subgraphs (???)

This has been proved for $t \leq 3$, and the 'if' part for $t \leq 4$.

\section{ADDED IN PROOF}

R. Pendavingh constructed graphs with $\lambda(G)>\mu(G)$, disproving one of the conjectures above.

\section{REFERENCES}

[1] R. Bacher, Y. Colin de Verdière, Multiplicités des valeurs propres et transformations étoiletriangle des graphes, Bulletin de la Société Mathématique de France 123 (1995) 101-117. MR 96k:05129

[2] E.G. Bajmóczy, I. Bárány, On a common generalization of Borsuk's and Radon's theorem, Acta Mathematica Academiae Scientiarum Hungaricae 34 (1979) 347-350. MR 84g:52007

[3] T. Böhme, On spatial representations of graphs, in: Contemporary Methods in Graph Theory (R. Bodendieck, ed.), BI-Wiss.-Verl. Mannheim, Wien/Zurich, 1990, pp. 151-167. MR 93a:05053

[4] Y. Colin de Verdière, Sur un nouvel invariant des graphes et un critère de planarité, Journal of Combinatorial Theory, Series B 50 (1990) 11-21. MR 91m:05068

[5] Y. Colin de Verdière, On a new graph invariant and a criterion for planarity, in: Graph Structure Theory (N. Robertson, P. Seymour, eds.), vol. 147, Contemporary Mathematics, American Mathematical Society, Providence, Rhode Island, 1993, pp. 137-147. MR 94h:05023

[6] H. van der Holst, A short proof of the planarity characterization of Colin de Verdière, Journal of Combinatorial Theory, Series B 65 (1995) 269-272. MR 96g:05050

[7] H. van der Holst, M. Laurent, A. Schrijver, On a minor-monotone graph invariant, Journal of Combinatorial Theory, Series B 65 (1995) 291-304. MR 96k:05096

[8] H. van der Holst, L. Lovász, A. Schrijver, On the invariance of Colin de Verdière's graph parameter under clique sums, Linear Algebra and Its Applications 226 (1995) 509-517. MR 97e:05113

[9] N. Robertson, P.D. Seymour, R. Thomas, A survey of linkless embeddings, in: Graph Structure Theory (N. Robertson, P. Seymour, eds.), Contemporary Mathematics, vol. 147, American Mathematical Society, Providence, Rhode Island, 1993, pp. 125-136. MR 94g:05026

[10] N. Robertson, P. Seymour, R. Thomas, Sachs' linkless embedding conjecture, Journal of Combinatorial Theory, Series B 64 (1995) 185-227. MR 96m:05072

[11] H. Saran, Constructive Results in Graph Minors: Linkless Embeddings, Ph.D. Thesis, University of California at Berkeley, 1989.

[12] K. Wagner, Über eine Eigenschaft der ebenen Komplexe, Mathematische Annalen 114 (1937) $570-590$.

Department of Computer Science, Yale University, New Haven, Connecticut 06520

E-mail address: lovasz@cs.yale.edu

CWi, Kruislaan 413, 1098 SJ Amsterdam, The Netherlands and Department of Mathematics, University of Amsterdam, Plantage Muidergracht 24, 1018 TV Amsterdam, The NETHERLANDS

E-mail address: lex@cwi.nl 